ГCOMMENTARY

Volume 13 Issue 22021

DOI: 10.21315/eimj2021.13.2.9

ARTICLE INFO

Received: 07-02-2021

Accepted: 17-03-2021

Online: 30-06-2021

\section{Common Myths About Entrustable Professional Activities}

\author{
Muhammad Zafar Iqbal', Mohamed M Al-Eraky² \\ ${ }^{1}$ Department of Medical Education, College of Medicine, Imam \\ Abdulrahman Bin Faisal University, Dammam, SAUDI ARABIA \\ ${ }^{2}$ Vice-President Office of Academic Initiatives, Imam Abdulrahman \\ Bin Faisal University, Dammam, SAUDI ARABIA
}

To cite this article: Iqbal MZ, Al-Eraky MM. Common myths about entrustable professional activities. Education in Medicine Journal. 2021;13(2):97-100. https://doi.org/10.21315/eimj2021 .13 .2 .9

To link to this article: https://doi.org/10.21315/eimj2021.13.2.9

\begin{abstract}
Entrustable professional activities (EPAs) are continuously gaining worldwide popularity in competency-based clinical training programmes. EPAs are discrete units of professional practice that are used to determine the readiness of a trainee in a particular training context. Despite their evergrowing recognition in health professions education, some critical misconceptions exist on the original nuance of EPAs that can potentially compromise their utility and operationalisation. For instance, EPAs are often confused with other educational entities such as competencies, outcomes and abilities. In this study, we attempt to address some common myths associated with EPAs and provide further clarification through examples to help readers differentiate between a true and a pseudo EPAs.
\end{abstract}

Keywords: Competency-based medical education, Entrustable professional activities, Competencies, Clinical training programmes, Entrustable tasks

Muhammad Zafar Iqbal, Office \# 3128, Third floor, Building A72, Department of Medical Education, College of Medicine, Imam Abdulrahman Bin Faisal University, Dammam, Saudi Arabia

Email: zafar@iau.edu.sa

\section{INTRODUCTION}

In 2005, ten Cate introduced the concept of entrustable professional activities (EPAs) to the health professions education community (1). Since then, much work has been done on EPAs at undergraduate and postgraduate levels and in various health professional domains (2-4). A salient reason for the popularity of EPAs in clinical training programmes is their ability to translate competencies into practice, thus filling the longstanding void in competencybased medical education (CBME) (5).
Despite their ever-growing recognition in health professions education, some critical misconceptions exist on the original nuance of EPAs that can potentially compromise their utility and operationalisation. These misunderstandings might also confuse those who wish to use EPAs to structure clinical training programmes in the future. Recently, scholars have also raised concerns against the imitative use of the term "EPAs" in practice. They have also considered pseudo EPAs a potential threat to the fundamental value of EPAs in advancing CBME (3). In addition, an ongoing confusion amongst 
educators exists where they often struggle to distinguish between EPAs, competencies and educational objectives (6). Considering the gravity of this situation, it is imperative to explicitly debunk some common myths about EPAs, which has remained a gap in the literature so far. In this commentary, we aim to address some common myths and provide further clarification through examples so that the researchers, programme directors and general readers can comprehend their actual concept.

\section{WHAT ARE EPAs?}

EPAs are professional tasks that can be entrusted to a health professional trainee to perform unsupervised after demonstrating sufficient proficiency in it (7). For any professional task to qualify as an EPA, it should be independent of other EPAs, observable in process and measurable in outcome. Moreover, it should be specific, focused and should have a clear beginning and end (7-8). The entrustable tasks must be suitable for credentialling and can only be performed by trained professionals. These tasks are then observed by the supervisors to define the readiness of a trainee in a specific context. In general, EPAs are broad in design; however, not all EPAs have the same breadth. The scope of practice of an EPA is usually determined by the level and specialty of training $(3,8)$. Before moving forward, please take a look at Table 1 and rationalise which one is an actual EPA in view of the above criteria.

\section{EPAs ARE NOT COMPETENCIES}

A common misconception is that an EPA is merely a modern term that has been coined to replace competencies $(2,6)$. Consequently, EPAs and competencies are often perceived as two sides of the same coin. Concurring to this misconception, Broussenko and colleagues found that almost $50 \%$ of their educator participants were unable to distinguish between EPAs and competencies (6). In reality, the fundamental purpose of EPAs is to help in translating theoretical competencies into practice instead of replacing them (9). An EPA refers to a professional work that is person neutral. It means that an EPA focuses on the task that must be performed in a healthcare setting regardless of who is performing it. Contrarily, a competency is the ability of a health professional that integrates knowledge, skills and attitudes (10). Therefore, an EPA is a professional activity (person neutral) that will require the acquisition, integration and application of multiple competencies (person specific) for its execution (11).

In Table 1, effective communication is not an EPA but a competency that will be required to perform entrustable tasks like breaking bad news or taking history.

Table 1: Which one is an actual EPA?

\begin{tabular}{ll}
\hline Example 1 & Effective communication. \\
Example 2 & Secures an intravenous line without complication during simulation training. \\
Example 3 & Palpates the liver of a jaundice patient during a bedside teaching session. \\
Example 4 & Skilfully performing a physical examination. \\
Example 5 & Performing lumbar punctures. \\
Example 6 & Providing care to a patient presented in an emergency room. \\
Example 7 & Counselling a patient with uncontrolled diabetes. \\
Example 8 & Contributing to community service to improve healthcare delivery system. \\
Example 9 & Brainstorming with healthcare team to devise a management plan. \\
Example 10 & Developing a professional portfolio during clinical residency. \\
\hline
\end{tabular}




\section{EPAs ARE NOT EDUCATIONAL OBJECTIVES}

Another common misunderstanding is that EPAs are designed to replace educational objectives or learning outcomes. Educational objectives provide specifications of instructional intent at a lower educational level and outcomes outline the intended ability of the learners by the end of an educational experience (12). Educational objectives or learning outcomes are usually designed for a lecture, PBL session, a bedside teaching session, or even for a course. Whereas, EPAs are holistic tasks that are usually broad in design and comprehensive enough to address a particular domain of the profession (3).

In view of the above argument, Examples 2 and 3 are not EPAs because both of them have two fundamental issues. First, they project the ability of a healthcare provider; second, they are designed for a specific teaching session which restricts their scope of practice. Example 2 can be transformed into an EPA by rephrasing it as "Securing an intravenous line without complication". Similarly, Example 3 (palpating the liver during examination) could be seen as an essential but smaller task that can be easily nested under a broader entrustable task (performing a physical examination). The nesting of small EPAs under a broad, overarching EPA is usually done to facilitate trainees and supervisors. These nested EPAs are not meant to be entrusted independently but to serve as a guide to the trainees as well as the supervisors to conceptualise the scope of practice of the broader EPA (13).

\section{EPAs ARE NOT PERSONAL OR PROFESSIONAL ABILITIES}

Another common error while designing EPAs is assigning an adjective to the task. Adjectives usually define the proficiency level of the person which defies the person neutrality of EPAs. As explained before, EPAs are merely professional tasks and are not meant to represent the proficiency or ability level of a trainee (11).

Although performing a physical examination (Example 4) is truly an EPA, the addition of the adjective "skilfully" in Example 4 takes away the sense of neutrality from the task by associating expertise level to it.

Examples 5, 6 and 7 are true EPAs, while the rest are not EPAs (or can be called as pseudo EPAs) as they do not fully concur with the essential features of EPAs. By using a checklist, Table 2 indicates the missing essential features in Examples 8, 9 and 10 that restricted us from labelling them as EPAs. The readers could use the same checklist as a lens to differentiate between a true and a pseudo EPAs.

Table 2: Incomplete EPAs with indication of missing essential features

\begin{tabular}{lccccc}
\hline Pseudo EPAs & Independent & Discrete & $\begin{array}{c}\text { Clear } \\
\text { beginning } \\
\text { and end }\end{array}$ & Observable & Measurable \\
\hline $\begin{array}{l}\text { Example 8: Contributing in } \\
\text { community service to improve } \\
\text { healthcare delivery system. }\end{array}$ & $\sqrt{ }$ & $\mathbf{n}$ & $\sqrt{ }$ & $\mathbf{x}$ & $\mathbf{x}$ \\
$\begin{array}{l}\text { Example 9: Brainstorming with } \\
\text { healthcare team to devise a } \\
\text { management plan. }\end{array}$ & $\sqrt{ }$ & $\times$ & $\times$ & $\times$ & $\mathbf{x}$ \\
$\begin{array}{l}\text { Example 10: Developing a } \\
\text { professional portfolio during } \\
\text { clinical residency. }\end{array}$ & $\sqrt{ }$ & $\sqrt{ }$ & $\times$ & $\times$ & $\mathbf{x}$ \\
\hline
\end{tabular}

Note: (x) Represents missing features. 


\section{CONCLUSION}

In this commentary, we have tried to bust some common myths associated with EPAs, based on theoretical rationale and supported by practical examples. We have disentangled the term EPAs from other education entities, such as competencies, outcomes and abilities. Furthermore, we stress upon the notion that EPAs are discrete tasks that are independent, observable in process and measurable in outcome. If a task lacks one or more hallmark features, then it should not be treated as an EPA. We concur with the proverbial saying, "All that glitters is not gold". Hopefully, our perspective will help the readers to differentiate between a true and a pseudo EPAs.

\section{REFERENCES}

1. ten Cate O. Entrustability of professional activities and competency-based training. Med Educ. 2005;39(12):1176-7. https://doi. org//10.1111/j.1365-2929.2005.02341.x

2. Shorey S, Lau TC, Lau ST, Ang E. Entrustable professional activities in health care education: a scoping review. Med Educ. 2019;53(8):766-77. https://doi.org/10.1111/ medu.13879

3. ten Cate O, Taylor DR. The recommended description of an entrustable professional activity: AMEE guide no. 140. Med Teach. 2020 November 9;1-9. https://doi.org/10 $.1080 / 0142159 X .2020 .1838465$

4. Iqbal $M Z$, Könings $\mathrm{KD}$, Al-Eraky $M$, AlSheikh MH, van Merriënboer JJG. Development of an entrustable professional activities (EPAs) framework for small group facilitators through a participatory design approach. Med Educ Online. 2020;25(1):1694309. https://doi.org/10.108 0/10872981.2019.1694309

5. ten Cate O, Billett S. Competency-based medical education: origins, perspectives and potentialities. Med Educ. 2014;48(3):32532. https://doi.org/10.1111/medu.12355
6. Broussenko M, Burns S, Leung F-H, Toubassi D. Can teachers distinguish competencies from entrustable professional activities? PRiMER. 2019;3:1-8. https://doi .org/10.22454/PRiMER.2019.433736

7. ten Cate O. Nuts and bolts of entrustable professional activities. J Grad Med Educ. 2013;5(1):157-8. https://doi.org/10.4300/ JGME-D-12-00380.1

8. ten Cate O, Chen HC, Hoff RG, Peters $\mathrm{H}$, Bok $\mathrm{H}$, van der Schaaf M. Curriculum development for the workplace using Entrustable Professional Activities (EPAs): AMEE guide no. 99. Med Teach. 2015;37(11):983-1002. https://doi.org/10 $.3109 / 0142159$ X.2015.1060308

9. ten Cate O. Competency-based education, entrustable professional activities, and the power of language. J Grad Med Educ. 2013;5(1):6-7. https://doi.org/10.4300/ JGME-D-12-00381.1

10. Frank JR, Snell LS, ten Cate O, Holmboe ES, Carraccio C, Swing $\mathrm{SR}$, et al. Competency-based medical education: theory to practice. Med Teach. 2010;32(8):638-45. https://doi.org/10.3109/ 0142159X.2010.501190

11. ten Cate O, Pool IA. The viability of interprofessional entrustable professional activities. Adv Heal Sci Educ. 2019;25(5):1255-62. https://doi.org/10 .1007/s10459-019-09950-0

12. Harden RM. Learning outcomes and instructional objectives: is there a difference? Med Teach. 2002;24(2):151-5. https://doi. org/10.1080/0142159022020687

13. Iqbal $M Z$, Könings $K D$, Al-Eraky $M$, van Merriënboer JJG. Entrustable professional activities for small-group facilitation: a validation study using modified Delphi technique. Teach Learn Med. 2021:1-10. https://doi.org/10.1080/10401334.2021.187 7714 